\title{
Comparative Analysis of Two Generalized Methodologies for Circuit Optimization
}

\author{
Alexander Zemliak, Fernando Reyes, Sergio Vergara, and Olga Felix
}

\begin{abstract}
The design process for analog network design is formulated on the basis of the optimum control theory. The artificially introduced special control vector is defined for the redistribution of computational costs between network analysis and parametric optimization. This redistribution minimizes computer time. The problem of the minimal-time network design can be formulated in this case as a classical problem of the optimal control for some functional minimization. There is a principal difference between the new approach and before elaborated methodology. This difference is based on a higher level of the problem generalization. In this case the structural basis of design strategies is more complete and this circumstance gives possibility to obtain a great value of computer time gain. Numerical results demonstrate the effectiveness and prospects of a more generalized approach to circuit optimization.
\end{abstract}

Keywords-Time-optimal design algorithm, control theory formulation, general methodology.

\section{INTRODUCTION}

$\mathrm{O}^{2}$ $\mathrm{NE}$ of the sources of overall improvement in design quality is the reduction of CPU time in the design of large systems. This problem has a great significance because it has a lot of applications, for example on VLSI electronic circuit design. Any traditional system design strategy includes two main parts: the mathematical model of the physical system that can be defined by the algebraic equations or differentialintegral equations and optimization procedure that achieves the optimum point of the design objective function. Within the framework of this concept, it is possible to change the optimization strategy and use different models and different analysis methods, but at each stage of the optimization process of the circuit there is a fixed number of equations of the mathematical model and a fixed number of independent parameters when optimizing the circuit.

Some powerful techniques have been used to reduce the

A. Zemliak is with the Department of Electronics, Autonomous University of Puebla, Puebla, 72570, Mexico and with the National Technical University of Ukraine, Kiev, 03056, Ukraine (phone: 52-222-229-5500; fax: 52-222229-5636; e-mail: azemliak@ yahoo.com).

F. Reyes is with the Department of Electronics, Autonomous University of Puebla, Puebla, 72570, Mexico (e-mail: ref62@gmail.com).

S. Vergara is with the Department of Electronics, Autonomous University of Puebla, Puebla, 72570, Mexico (e-mail: svergara@ece.buap.mx).

O. Felix is with the Department of Electronics, Autonomous University of Puebla, Puebla, 72570, Mexico (e-mail: olgafelixbeltran@gmail.com). time required to analyze the circuit. Because a matrix of the large-scale circuit is a very sparse, the special sparse matrix techniques are used successfully for this purpose [1-2]. Other approach to reduce the amount of computational required for both linear and nonlinear equations is based on the decomposition techniques. The partitioning of a circuit matrix into bordered-block diagonal form can be done by branches tearing as in [3], or by nodes tearing as in [4] and jointly with direct solution, algorithms gives the solution of the problem. The extension of the direct solution methods can be obtained by hierarchical decomposition and macro model representation [5]. Other approach for achieving decomposition at the nonlinear level consists on a special iteration techniques and has been realized in [6] for the iterated timing analysis and circuit simulation. Optimization technique that is used for the circuit optimization and design, exert a very strong influence on the total necessary computer time too. The numerical methods are developed both for the unconstrained and for the constrained optimization [7] and will be improved later on. The practical aspects of these methods were developed for the electronic circuits design with the different optimization criterions [8-9]. The fundamental problems of the development, structure elaboration, and adaptation of the automation design systems have been examine in some papers [10-11].

The ideas of designing the system described above can be called the traditional approach or the traditional strategy, since the method of analysis is based on the laws of Kirchhoff.

Other idea for the problem of optimizing the circuit were developed at a heuristic level several decades ago [12]. This idea was based on the Kirchhoff laws ignoring for all the circuit or for the circuit part. The special cost function is minimized instead of the circuit equation solving. This idea was developed in practical aspect for the microwave circuit optimization [13] and for the synthesis of high-performance analog circuits [14] in extremely case, when the total system model was eliminated. The authors of the last papers affirm that the design time was reduced significantly. This last idea can be named as the modified traditional design strategy.

At the same time, all these ideas can be generalized to reduce the total computer design time for system design. This generalization can be done on the basis of the control theory approach and includes the special control function to control 
the design process. This approach consists of the reformulation of the total design problem and generalization of it to obtain a set of different design strategies inside the same optimization procedure [15]. The number of the different design strategies, which appear in the generalized theory, is equal to $2^{M}$ for the constant value of all the control functions, where $M$ is the number of dependent parameters. These strategies serve as the structural basis for more strategies construction with the variable control functions. The main problem of this new formulation is the unknown optimal dependency of the control function vector that satisfies to the time-optimal design algorithm. One way to solve this problem is to use the Lyapunov function of the design process [16].

However, the developed theory [15] is not the most general. In the limits of this approach only initially dependent system parameters can be transformed to the independent but the inverse transformation is not supposed. The next more general approach for the system design supposes that initially independent and dependent system parameters are completely equal in rights, i.e. any system parameter can be defined as independent or dependent one. In this case we have more vast set of the design strategies that compose the structural basis and more possibility to the optimal design strategy construct.

\section{PROBLEM FORMULATION}

In accordance with the new system design methodology [15] the design process can be defined as the problem of the cost function $C(X)$ minimization for $X \in R^{N}$ by the optimization procedure and by the analysis of the modified electronic system model. The optimization procedure can be determined in continuous form as:

$$
\frac{d x_{i}}{d t}=f_{i}(X, U), \quad i=1,2, \ldots, N
$$

The modified electronic system model can be expressed in the next form:

$$
\left(1-u_{j}\right) g_{j}(X)=0, \quad j=1,2, \ldots, M
$$

where $N=K+M, K$ is the number of independent system parameters, $M$ is the number of dependent system parameters, $X$ is the vector of all variables $X=\left(x_{1}, x_{2}, \ldots, x_{K}, x_{K+1}, x_{K+2}, \ldots, x_{N}\right) ; U$ is the vector of control variables $U=\left(u_{1}, u_{2}, \ldots, u_{M}\right) ; u_{j} \in \Omega ; \Omega=\{0 ; 1\}$. The functions of the right hand part of the system (1) depend on the concrete optimization algorithm and, for instance, for the gradient method are determined as:

$$
f_{i}(X, U)=-b \frac{\delta}{\delta x_{i}}\left\{C(X)+\frac{1}{\varepsilon} \sum_{j=1}^{M} u_{j} g_{j}^{2}(X)\right\}
$$

for $i=1,2, \ldots, K$,

$$
\begin{aligned}
f_{i}(X, U)= & -b \cdot u_{i-K} \frac{\delta}{\delta x_{i}}\left\{C(X)+\frac{1}{\varepsilon} \sum_{j=1}^{M} u_{j} g_{j}^{2}(X)\right\} \\
& +\frac{\left(1-u_{i-K}\right)}{d t}\left\{-x_{i}^{\prime}+\eta_{i}(X)\right\}
\end{aligned}
$$

for $i=K+1, K+2, \ldots, N$,

where $b$ is the iteration parameter; the operator $\delta / \delta x_{i}$ hear and below means $\frac{\delta}{\delta x_{i}} \varphi(X)=\frac{\partial \varphi(X)}{\partial x_{i}}+\sum_{p=K+1}^{K+M} \frac{\partial \varphi(X)}{\partial x_{p}} \frac{\partial x_{p}}{\partial x_{i}}$, $x_{i}^{\prime}$ is equal to $x_{i}(t-d t) ; \quad \eta_{i}(X)$ is the implicit function $\left(x_{i}=\eta_{i}(X)\right)$ that is determined by the system $(2), C(X)$ is the cost function of the design process.

The task of searching for an optimal design algorithm is currently defined as a typical problem of control theory to minimize some functional. The total computer design time serves as the necessary functional in this case. An optimal or quasi-optimal solution to a design problem can be obtained based on different analytical [17] or numerical [18-21] methods.

In the above formulation, the initially dependent parameters that defined for $i=K+1, K+2, \ldots, N$, can be transformed into independent ones when $u_{j}=1$ and it is dependent when $u_{j}=0$.

On the other hand the initially independent parameters that can be defined for $i=1,2, \ldots, K$, are independent ones always.

In this article, we have developed a new approach that allows us to obtain a greater generalization than in the methodology described above. We suppose now that all of the system parameters can be independent or dependent ones. In this case we need to change the equation (2) for the system model definition and the equation (3) for the right parts description.

The equation (2) defines the system model and is transformed now to the next one:

$$
\begin{gathered}
\left(1-u_{i}\right) g_{j}(X)=0 \\
i=1,2, \ldots, N \text { and } j \in J
\end{gathered}
$$

where $J$ is the index set for all those functions $g_{j}(X)$ for which $u_{i}=0, J=\left\{j_{1}, j_{2}, \ldots, j_{z}\right\}, j_{s} \in \Pi$ with $s=1,2, \ldots, Z$, $\Pi$ is the set of the indexes from 1 to $M, \Pi=\{1,2, \ldots, M\}, Z$ is the number of the equations that will be left in the system (4), $Z \in\{0,1 \ldots, M\}$.

The right hand side of the system (1) is defined now as:

$$
\begin{aligned}
f_{i}(X, U)= & -b \cdot u_{i} \frac{\delta}{\delta x_{i}} F(X, U) \\
& +\frac{\left(1-u_{i}\right)}{d t}\left\{-x_{i}(t-d t)+\eta(X)\right\}
\end{aligned}
$$

for $\quad i=1,2, \ldots, N$, 
where $F(X, U)$ is the generalized objective function and it is defined as:

$$
F(X, U)=C(X)+\frac{1}{\varepsilon} \sum_{j \in \Pi \backslash J} g_{j}^{2}(X)
$$

This new definition of the design process is more general than in [15]. It generalizes the methodology for the system design and produces more representative structural basis of different design strategies. The total number of the different design strategies, which compose the structural basis, is equal to $\sum_{i=0}^{M} C_{K+M}^{i}$. We expect the new possibilities to accelerate the design process in this case.

\section{NUMERICAL RESULTS}

New generalized methodology has been used for some nonlinear electronic circuit optimization. The numerical results correspond to the integration of the system (1) with variable optimized step. The cost function $C(X)$ has been defined as a sum of squares of differences between before defined and current value of some node voltages.

\section{A. Example 1}

The four-node circuit is analyzed below (Fig. 1) by means of the new generalized methodology.

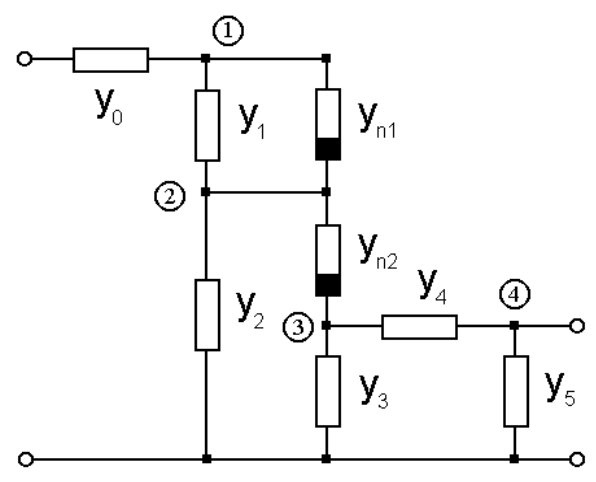

Fig. 1 Four-node circuit topology

The design problem includes five parameters as admittances $\left(x_{1}, x_{2}, x_{3}, x_{4}, x_{5}\right)$, where $x_{1}^{2}=y_{1}, \quad x_{2}^{2}=y_{2}, x_{3}^{2}=y_{3}$, $x_{4}^{2}=y_{4}, x_{5}^{2}=y_{5}$, and four parameters as nodal voltages $\left(x_{6}, x_{7}, x_{8}, x_{9}\right)$, where $x_{6}=V_{1}, x_{7}=V_{2}, x_{8}=V_{3}, x_{9}=V_{4}$, The nonlinear elements are defined as: $y_{n 1}=a_{n 1}+b_{n 1} \cdot\left(V_{1}-V_{2}\right)^{2}, \quad y_{n 2}=a_{n 2}+b_{n 2} \cdot\left(V_{2}-V_{3}\right)^{2} . \quad$ The control vector $U$ includes nine components $\left(u_{1}, u_{2}, \ldots, u_{9}\right)$. The model of circuit (4) includes 4 equations and functions $g_{j}(X)$ are defined by (7):

$$
\begin{gathered}
g_{1}(X) \equiv y_{0}\left(V_{0}-x_{6}\right)-\left[x_{1}^{2}+a_{n 1}+b_{n 1}\left(x_{6}-x_{7}\right)^{2}\right]\left(x_{6}-x_{7}\right)=0 \\
g_{2}(X) \equiv\left[x_{1}^{2}+a_{n 1}+b_{n 1}\left(x_{6}-x_{7}\right)^{2}\right]\left(x_{6}-x_{7}\right) \\
-x_{2}^{2} x_{7}-\left[a_{n 2}+b_{n 2}\left(x_{7}-x_{8}\right)^{2}\right]\left(x_{7}-x_{8}\right)=0 \\
g_{3}(X) \equiv\left[a_{n 2}+b_{n 2}\left(x_{7}-x_{8}\right)^{2}\right]\left(x_{7}-x_{8}\right) \\
-\left(x_{3}^{2}+x_{4}^{2}\right) x_{8}-x_{4}^{2} x_{9}=0 \\
g_{4}(X) \equiv x_{4}^{2} x_{8}-\left(x_{4}^{2}+x_{5}^{2}\right) x_{9}=0
\end{gathered}
$$

The optimization procedure (1) includes nine equations. The cost function $C(X)$ of the design process is defined by the following form: $C(X)=\left(x_{9}-k_{0}\right)^{2}+\left(x_{6}-x_{7}-k_{1}\right)^{2}+\left(x_{7}-x_{8}-k_{2}\right)^{2}$.

The total number of the different design strategies that compose the structural basis of the generalized theory is equal $\sum_{i=0}^{4} C_{9}^{i}=256$. At the same time the structural basis of the

\begin{tabular}{|c|c|c|c|}
\hline \multirow{3}{*}{\begin{tabular}{|l|} 
\\
\end{tabular}} & \multirow{2}{*}{$\begin{array}{l}\text { Control functions } \\
\text { vector } \\
\text { U (u1, u2, } 43, \mathrm{u4}, \mathrm{u} 5, \mathrm{u6}, \mathrm{u} 7, \mathrm{u8}, \mathrm{ug})\end{array}$} & \multicolumn{2}{|c|}{\begin{tabular}{|l} 
Calculation results \\
\end{tabular}} \\
\hline & & $\begin{array}{l}\text { Iterations } \\
\text { number }\end{array}$ & \begin{tabular}{|l|} 
Total design \\
time (sec)
\end{tabular} \\
\hline & $(111010001)$ & 5 & \begin{tabular}{|l|}
0.0031 \\
\end{tabular} \\
\hline 9 & $(111110001)$ & 397 & 0.4312 \\
\hline 3 & $(111011001)$ & 5 & 0.0029 \\
\hline 4 & $(110111110)$ & 119 & 0.0209 \\
\hline 5 & $(111100101)$ & 101 & 0.0232 \\
\hline 6 & $(111010011)$ & 15 & 0.0134 \\
\hline 7 & $(111011101)$ & 5 & 0.0009 \\
\hline 8 & $(111011111)$ & 101 & 0.0243 \\
\hline 9 & $(111100111)$ & 185 & 0.0324 \\
\hline 10 & $(111101001)$ & 74 & 0.0102 \\
\hline 11 & $(111101011)$ & 121 & 0.0254 \\
\hline 12 & $(111101111)$ & 159 & 0.0127 \\
\hline 13 & $(111$ & 33 & 0.0263 \\
\hline 14 & $(111110001)$ & 397 & 0.4317 \\
\hline \begin{tabular}{|l|}
15 \\
\end{tabular} & $(111110010)$ & 6548 & 7.1392 \\
\hline \begin{tabular}{|l|}
16 \\
\end{tabular} & $(111110011)$ & 76 & 0.0122 \\
\hline \begin{tabular}{|l|}
17 \\
\end{tabular} & $(111110100)$ & 456 & 0.5113 \\
\hline \begin{tabular}{|c|}
18 \\
\end{tabular} & $(111$ & 24 & \\
\hline \begin{tabular}{|l|}
19 \\
\end{tabular} & $(111110110)$ & 3750 & 4.3661 \\
\hline 20 & $(111110111)$ & 90 & 0.0095 \\
\hline \begin{tabular}{|l|}
21 \\
\end{tabular} & $(111111000)$ & 68 & 0.0354 \\
\hline \begin{tabular}{|l|l|}
22 \\
\end{tabular} & $\frac{(111111001)}{(11)}$ & 596 & 0.6213 \\
\hline \begin{tabular}{|l|l|}
23 \\
\end{tabular} & $(111111010)$ & 5408 & 6.2191 \\
\hline \begin{tabular}{|l|}
24 \\
\end{tabular} & $(111111011)$ & 78 & 0.0255 \\
\hline \begin{tabular}{|l|l|}
25 \\
\end{tabular} & $(111111100)$ & 238 & 0.2104 \\
\hline \begin{tabular}{|l|}
26 \\
\end{tabular} & $(111111101)$ & 77 & 0.0227 \\
\hline \begin{tabular}{|l|}
27 \\
\end{tabular} & $(1111$ & 139 & 0.0131 \\
\hline \begin{tabular}{|l|}
28 \\
\end{tabular} & $(111111111)$ & 131 & 0.0103 \\
\hline
\end{tabular}
previous developed theory includes 16 strategies only $\left(2^{4}\right)$. The results of the analysis of some strategies of structural basis that include all the "old" strategies (the last 16 strategies) and some new strategies (from 1 to 12 ) are shown in Table 1.

Table 1. Some strategies of structural basis for four-node circuit 
Strategy 13 corresponds to the Traditional Design Strategy (TDS). There are seven different strategies in the "old" group that have the design time less that the TDS. These are the strategies $16,18,20,24,26,27$ and 28 . The strategy 18 is the optimal one among all of the "old" strategies and it has the time gain 5.06 with respect to the TDS. On the other hand the best strategy among all the strategies (number 7) of the Table 2 has the time gain 29.2. So, we have an additional acceleration in 5.77 times. This effect was obtained due to the utilization of more extensive structural basis and it serves as the principal result of the new generalized methodology. It is clear that further optimization of the control vector $U$ can increase this time gain and in this case we can improve all the results.

\section{B. Example 2}

The next example corresponds to the three-stage transistor amplifier in Fig. 2.

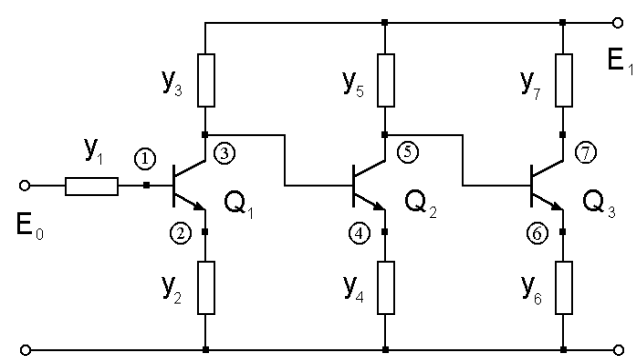

Fig. 2 Three-stage transistor amplifier

The Ebers-Moll static model of transistor has been used [22]. The cost function $C(X)$ is defined by the formula: $C(X)=\sum_{i=1}^{3}\left(I_{C i}-m_{i}\right)^{2}$, where $m_{i}$ is a given collector current for the transistor number $i$.

In this case the vector $X$ includes 14 components. Seven components define the independent parameters $x_{1}^{2}=y_{1}$, $x_{2}^{2}=y_{2}, x_{3}^{2}=y_{3}, x_{4}^{2}=y_{4}, x_{5}^{2}=y_{5}, x_{6}^{2}=y_{6}, x_{7}^{2}=y_{7}$ and other seven components $x_{8}=V_{1}, \quad x_{9}=V_{2}, \quad x_{10}=V_{3}, \quad x_{11}=V_{4}$, $x_{12}=V_{5}, x_{13}=V_{6}, x_{14}=V_{7}$ define the dependent parameters in accordance with the traditional approach. The cost function $C(X)$ for the optimization problem was defined by the formula similar to the previous example.

The structural basis consists of 128 different design strategies according to the first level of generalization. On the other hand the structural basis of the second level of generalization is equal to $\sum_{i=0}^{7} C_{14}^{i}=9908$. Once again we have very broadened structural basis in the second level of generalization. The results of the analysis of some design strategies for this network are presented in Table 2.

The design strategies numbered from 15 to 28 belong to the subset that appears in limits of the first level of design methodology generalization. The strategy 15 that corresponds to the control vector (11111110000000) is the traditional design strategy.
Table 2. Some strategies of the structural basis for three-stage transistor amplifier

\begin{tabular}{|c|c|c|c|}
\hline \multirow[t]{2}{*}{$\mathrm{N}$} & \multirow{2}{*}{$\begin{array}{l}\text { Control functions } \\
\text { vector } \\
\mathrm{U}(\mathrm{u} 1, \mathrm{u} 2, \ldots, \mathrm{u} 14)\end{array}$} & \multicolumn{2}{|c|}{ Calculation results } \\
\hline & & \begin{tabular}{|l|} 
Iterations \\
number
\end{tabular} & \begin{tabular}{|l} 
Total design \\
time $(\mathrm{sec})$
\end{tabular} \\
\hline & $(00000001111111)$ & 72 & 0.549 \\
\hline & $(00000011111111)$ & 235 & 1.030 \\
\hline 3 & $(00000111111111)$ & 506 & 1.031 \\
\hline 4 & $(00001111111111)$ & 891 & 2.980 \\
\hline & $(00011111111111)$ & 660 & 1.050 \\
\hline 6 & $(00111111111111)$ & 1262 & 2.002 \\
\hline 7 & $(01111111111111)$ & 504 & 0.953 \\
\hline 8 & $(10111111111111)$ & 351 & 0.380 \\
\hline$\overline{9}$ & $(11011111111111)$ & 316 & 0.350 \\
\hline 10 & $(1110$ & 662 & 0.709 \\
\hline 11 & $(11110111111111)$ & 801 & 0.986 \\
\hline 12 & $(11$ & 532 & 0.956 \\
\hline 13 & $(11)$ & 11993 & 129.003 \\
\hline 14 & $(11111$ & 308 & 0.030 \\
\hline 15 & $(11$ & 38775 & 351.456 \\
\hline 16 & $(111$ & 100843 & 742.993 \\
\hline 17 & $(11111110000100)$ & 45407 & 440.014 \\
\hline 18 & $3(11111110010000)$ & 2643 & 29.002 \\
\hline 19 & $(11111110100000)$ & 82811 & 1163.987 \\
\hline 20 & $(11111110111111)$ & 1127 & 1.020 \\
\hline 21 & $(11111111000000)$ & 10454 & 89.019 \\
\hline 22 & $(11111111011111)$ & 540 & 0.955 \\
\hline 23 & $(11111111101111)$ & 53880 & 61.040 \\
\hline 24 & $(11111111110111)$ & 1008 & 1.007 \\
\hline 25 & $(11111111111011)$ & 5647 & 6.012 \\
\hline 26 & $(11111111111101)$ & 226 & 1.885 \\
\hline 27 & $(11111111111110)$ & 7441 & 7.999 \\
\hline 28 & $(11111111111111)$ & 3979 & 2.005 \\
\hline
\end{tabular}

The strategy 22 that corresponds to the control vector (11111111011111) has the minimum computer time among all the strategies of this subset. The time gain in this case is equal to 368 times. The strategies from 1 to 14 belong to the subset of new design strategies. Six strategies of this subset have the design time lesser than the best strategy of the "old" structural basis. The best strategy among new structural basis has the time gain 11715 times with respect to the traditional design strategy and has an additional time gain 31.8 times with respect to the better "old" strategy.

\section{Example 3}

The last example corresponds to the transistor amplifier in Fig. 3. In this case the vector $X$ includes 13 components.

Five components define the independent parameters $x_{1}^{2}=y_{1}$, $x_{2}^{2}=y_{2}, x_{3}^{2}=y_{3}, x_{4}^{2}=y_{4}, x_{5}^{2}=y_{5}$ and other eight components $x_{6}=V_{1}, x_{7}=V_{2}, x_{8}=V_{3}, x_{9}=V_{4}, x_{10}=V_{5}, x_{11}=V_{6}, x_{12}=V_{7}$, $x_{13}=V_{8}$ define the dependent parameters in accordance with the traditional approach. The cost function $C(X)$ for the design problem was defined by the formula similar to the previous example. The structural basis consists of 256 different design strategies according to the first level of generalization.

On the other hand the structural basis of the second level of generalization is equal to $\sum_{i=0}^{8} C_{13}^{i}=7099$. 


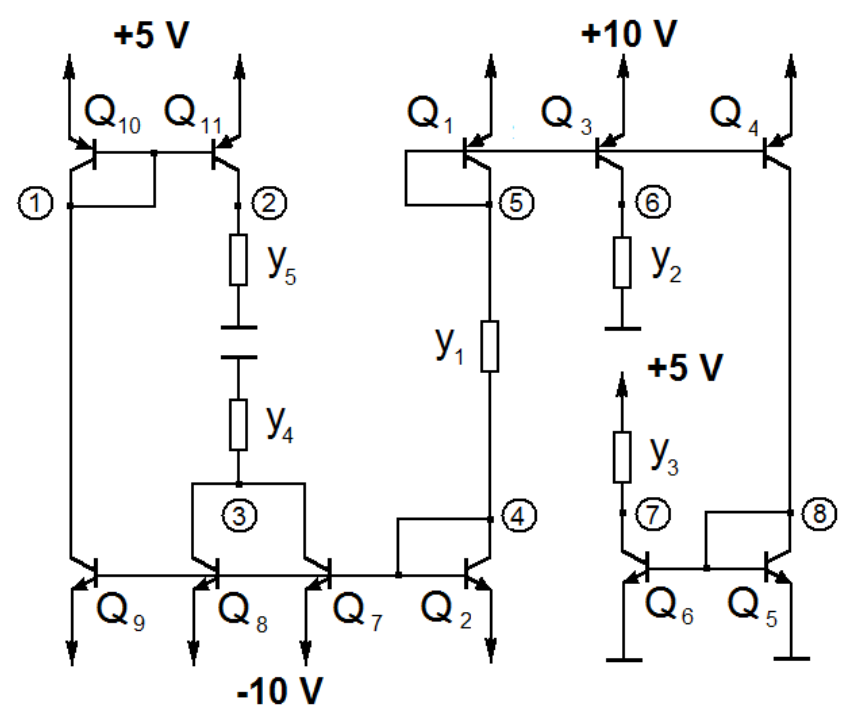

Fig. 3 Eight-node transistor amplifier

Once again we have very broadened structural basis in the second case. The results of the analysis of TDS and some strategies that have the design time less than TDS for this network are presented in Table 3 .

The design strategies numbered from 22 to 45 belong to the subset that appears on the basis of the first level of design methodology generalization. The strategy 22 that corresponds to the control vector (1111100000000) is the TDS. This strategy has a large number of iteration steps and a large computer time $(24.75 \mathrm{sec})$. Other strategies that are presented in this table have considerably less iteration number and computer time. For instance the MTDS with control vector (1111111111111) has computer time $0.202 \mathrm{sec}$. The time gain is equal to 123.7 times. The strategy 34 that corresponds to the control vector (1111111011110) has the minimum computer time among all the strategies of this subset. The time gain is equal to 1295 times.

The strategies from 1 to 21 belong to the subset of new design strategies. Strategy 18 of this subset has the design time lesser than the best strategy of the "old" structural basis. This strategy belong to the new structural basis and it has the time gain 1447 times with respect to the traditional design strategy and has an additional time gain 1.12 times with respect to the better strategy of the first level of the generalization.

Moreover among the "old" strategies there are 6 strategies that have the time gain more than 500 and 9 strategies that have the time gain more than 400 . On the other hand among the "new" strategies there are 11 that have the time gain more than 500 and 13 strategies that have the time gain more than 400 .

So, taking into consideration the obtained results we can state that the second level of the design methodology generalization gives the possibility to improve all characteristics of the generalized design theory.

Further analysis may be focused on the problem of the optimal design strategy searching by means of the control vector manipulation. It is intuitively clear that we can obtain very great time gain by means of the new structural basis.
Table 3. Some strategies of the structural basis for transistor amplifier in Fig. 3

\begin{tabular}{|c|c|c|c|}
\hline $\mathrm{N}$ & $\begin{array}{l}\text { Control functions } \\
\text { vector } \\
\mathrm{U}(\mathrm{u} 1, \mathrm{u} 2, \ldots, \mathrm{u} 13)\end{array}$ & \begin{tabular}{|l} 
Calculation \\
Iterations \\
number
\end{tabular} & $\begin{array}{l}\text { results } \\
\begin{array}{l}\text { Total design } \\
\text { time (sec) }\end{array}\end{array}$ \\
\hline 1 & $(0011111011111)$ & 131 & 0.0680 \\
\hline 2 & $(0011111111111)$ & 138 & 0.0477 \\
\hline 3 & $(0101111111111)$ & 118 & 0.0441 \\
\hline 4 & $(0110111111111)$ & 83 & 0.0343 \\
\hline 5 & $(0111011111111)$ & 142 & 0.0536 \\
\hline 6 & $(0111101111111)$ & 123 & 0.0464 \\
\hline 7 & $(0111111111111)$ & 155 & 0.0422 \\
\hline 8 & $(1001111111111)$ & 232 & 0.0754 \\
\hline 9 & $(1010111111111)$ & 338 & 0.0982 \\
\hline $\mid 10$ & $(1011011111111)$ & 247 & 0.0668 \\
\hline \begin{tabular}{|l|}
11 \\
\end{tabular} & $(1011101111111)$ & 145 & 0.0402 \\
\hline 12 & $(1011110111111)$ & 247 & 0.0657 \\
\hline $13 \mid$ & $(1011111011111)$ & 156 & 0.0478 \\
\hline 14 & $(1011111101111)$ & 502 & 0.1425 \\
\hline 15 & $(1011111110111)$ & 300 & 0.1145 \\
\hline 16 & $(1011111111101)$ & 287 & 0.0825 \\
\hline 17 & $(1011111111110)$ & 132 & 0.0425 \\
\hline 18 & $(1011111111111)$ & 77 & 0.0171 \\
\hline 19 & $(1110111111110)$ & 83 & 0.0248 \\
\hline 20 & $(1110111111111)$ & 254 & 0.0602 \\
\hline 21 & $(1111011111111)$ & 176 & 0.0339 \\
\hline 22 & $(1111100000000)$ & 6990 & 24.7500 \\
\hline 23 & $(1111100000001)$ & 90 & 0.1454 \\
\hline 24 & $(1111100000011)$ & 246 & 0.3410 \\
\hline 25 & $(1111100000111)$ & 203 & 0.2231 \\
\hline 26 & $(1111100001111)$ & 875 & 0.7300 \\
\hline 27 & $(1111100011111)$ & 299 & 0.1530 \\
\hline 28 & $(1111100111111)$ & 301 & 0.1210 \\
\hline 29 & $(1111110000001)$ & 159 & 0.2040 \\
\hline 30 & $(1111110001111)$ & 777 & 0.6000 \\
\hline 31 & $(1111110111110)$ & 89 & 0.0380 \\
\hline 32 & $(1111110111111)$ & 216 & 0.0611 \\
\hline 33 & $(1111111000001)$ & 157 & 0.1450 \\
\hline 34 & $(1111111011110)$ & 59 & 0.0191 \\
\hline 35 & $(1111111011111)$ & 153 & 0.0530 \\
\hline 36 & $(1111111101110)$ & 303 & 0.1100 \\
\hline 37 & $(1111111101111)$ & 379 & 0.0980 \\
\hline 38 & $(1111111110110)$ & 90 & 0.0420 \\
\hline 39 & $(1111111110111)$ & 190 & 0.0750 \\
\hline 40 & $(1111111111010)$ & 132 & 0.0361 \\
\hline 41 & $(1111111111011)$ & 207 & 0.0452 \\
\hline 42 & $(1111111111100)$ & 155 & 0.0571 \\
\hline 43 & $(1111111111101)$ & 257 & 0.0573 \\
\hline 44 & $(1111111111110)$ & 121 & 0.0350 \\
\hline 45 & $(1111111111111)$ & 607 & 0.0871 \\
\hline
\end{tabular}

\section{CONCLUSION}

The traditional approach for the analog circuit design is not time-optimal. The time-optimal design algorithm is formulated as the problem of the functional minimization of the optimal control theory. In this case it is necessary to select one optimal trajectory from the quasi-infinite number of the different design strategies, which are produced. The new approach to the electronic circuit design methodology has been developed now. This approach generates structural basis of the different design strategies that is more broadened than for the previous developed methodology. The total number of the different design strategies, which compose the structural basis by this approach, is equal to $\sum_{i=0}^{M} C_{K+M}^{i}$. This new structural basis 
serves as the necessary set for searching the optimal design strategy. This approach can reduce considerably the total computer time for the system optimization. Analysis of the different problems of the electronic system design shows a significant potential of the new level of generalized design methodology. The potential gain of computer time that can be obtain on the basis of new approach is significantly more than for the previous developed methodology.

\section{REFERENCES}

[1] J. R. Bunch and D.J. Rose, (Eds.), Sparse Matrix Computations, New York: Acad. Press, 1976.

[2] O. Osterby and Z. Zlatev, Direct Methods for Sparse Matrices, New York: Springer-Verlag, 1983.

[3] F. F. Wu, "Solution of Large-Scale Networks by Tearing", IEEE Trans. Circuits Syst., vol. CAS-23, no. 12, pp. 706-713, 1976.

[4] A. Sangiovanni-Vincentelli, L.K. Chen and L.O. Chua, "An Efficient Cluster Algorithm for Tearing Large-Scale Networks", IEEE Trans. Circ. Syst.,vol. 24, no. 12, pp. 709-717, 1977.

[5] N. Rabat, A. E. Ruehli, G. W. Mahoney and J. J. Coleman, "A Survey of Macromodeling", Proc. of the IEEE Int. Symp. C\&S, 1985, pp.139143.

[6] A. E. Ruehli, G. Ditlow, "Circuit Analysis, Logic Simulation and Design Verification for VLSI", Proc. IEEE, vol. 71, no. 1, pp. 36-68, 1983.

[7] R. Fletcher, Practical Methods of Optimization, New York: John Wiley and Sons, vol. 1, 1980, vol. 2, 1981.

[8] R. K. Brayton, G. D. Hachtel and A. L. Sangiovanni-Vincentelli, "A survey of optimization techniques for integrated-circuit design", Proc. IEEE, vol. 69, pp. 1334-1362, 1981.

[9] R. E. Massara, Optimization Methods in Electronic Circuit Design, Harlow: Longman Scientific \& Technical, 1991.

[10] A. I. Petrenko, "The Complexity and Adaptation of the Modern Design Automation Systems", Izvestiya VUZ Radioelectronics, vol. 31, no. 6, pp. 27-31, 1988.

[11] I. P. Norenkov, "The Structure Development of the Design Automation Systems", Izvestiya VUZ Radioelectronics, vol. 32, no. 6, pp. 25-29, 1989.

[12] I. S. Kashirsky and I. K. Trokhimenko, The Generalized Optimization of Electronic Circuits, Kiev: Tekhnika, 1979.

[13] V. Rizzoli, A. Costanzo and C. Cecchetti, "Numerical optimization of broadband nonlinear microwave circuits", IEEE MTT-S Int. Symp., Vol. 1, 1990, pp. 335-338.

[14] E. S. Ochotta, R. A. Rutenbar and L. R. Carley, "Synthesis of HighPerformance Analog Circuits in ASTRX/OBLX", IEEE Trans. on $C A D$, vol. 15, no. 3, pp. 273-294, 1996.

[15] A. M. Zemliak, "Design of Analog Networks by Control Theory Methods, Part 1, Theory", Radioelectronics and Communications Systems, vol. 47, no. 5, pp. 11-17, 2004.

[16] A. Zemliak and T. Markina, "Behaviour of Lyapunov's function for different strategies of circuit optimization", International Journal of Electronics, vol. 102, no. 4, pp. 619-634, 2015.

[17] L. S. Pontryagin, V. G. Boltyanskii, R. V. Gamkrelidze and E. F. Mishchenko, The Mathematical Theory of Optimal Processes, New York: Interscience Publishers, Inc., 1962.

[18] J. B. Rosen, "Iterative Solution of Nonlinear Optimal Control Problems", J. SIAM, Control Series A, pp. 223-244, 1966.

[19] I. A. Krylov and F. L. Chernousko, Consecutive Approximation Algorithm for Optimal Control Problems, J. of Numer. Math. and Math. Pfysics, vol. 12, № 1, 1972, pp. 14-34.

[20] R. P. Fedorenko, Approximate Solution of Optimal Control Problems, Moscow: Nauka, 1978.

[21] R. Pytlak, Numerical Methods for Optimal Control Problems with State Constraints, Springer-Verlag, Berlin, 1999.

[22] G. Massobrio and P. Antognetti, Semiconductor Device Modeling with SPICE, New York: Mc. Graw-Hill, Inc., 1993.
Alexander Zemliak is currently a Professor of Department of Physics and Mathematics, Autonomous University of Puebla. He is the author of over 300 publications in peer-reviewed journals and conference proceedings. His research interests are related to systems analysis, optimization and design. Prof. Zemliak is a member of Ukrainian Scientific Society, National System of Investigators of Mexico, Senior Member of IEEE, member of IEICE and WSEAS.

Fernando Reyes is currently a Professor of Department of Electronics, Autonomous University of Puebla. His research interests are in automation and robotics. He has authored of three books, and over 60 papers in refereed journals and conference proceedings. Prof. Reyes is a member of National System of Investigators of Mexico.

Sergio Vergara is currently a Professor of Department of Electronics, Autonomous University of Puebla. His research interests are in automation and robotics. He is the author of over 50 publications in peer-reviewed journals and conference proceedings. Prof. Vergara is a member of National System of Investigators of Mexico.

Olga Felix is currently a Professor of Department of Electronics, Autonomous University of Puebla, Mexico. Her research interests are in quantum physics, automation and robotics. She is the author of over 40 publications in peer-reviewed journals and conference proceedings. Prof. Felix is a member of National System of Investigators of Mexico. 